\title{
Video Article \\ A Protocol for Rapid Post-mortem Cell Culture of Diffuse Intrinsic Pontine Glioma (DIPG)
}

\author{
Grant L. Lin ${ }^{1}$, Michelle Monje ${ }^{2}$ \\ ${ }^{1}$ Graduate Program in Neuroscience, Department of Neurology, Institute for Stem Cell Biology and Regenerative Medicine, Stanford University School of Medicine

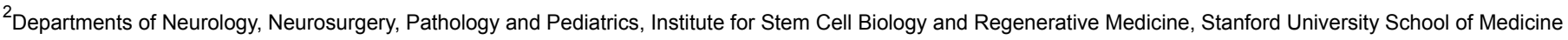

Correspondence to: Michelle Monje at mmonje@stanford.edu

URL: https://www.jove.com/video/55360

DOI: doi: $10.3791 / 55360$

Keywords: Cancer Research, Issue 121, DIPG, glioma, patient-derived cell culture, rapid autopsy, brain, tissue dissociation, cancer

Date Published: $3 / 7 / 2017$

Citation: Lin, G.L., Monje, M. A Protocol for Rapid Post-mortem Cell Culture of Diffuse Intrinsic Pontine Glioma (DIPG). J. Vis. Exp. (121), e55360, doi:10.3791/55360 (2017).

\section{Abstract}

Diffuse Intrinsic Pontine Glioma (DIPG) is a childhood brainstem tumor that carries a universally fatal prognosis. Because surgical resection is not a viable treatment strategy and biopsy is not routinely performed, the availability of patient samples for research is limited. Consequently, efforts to study this disease have been challenged by a paucity of faithful disease models. To address this need, we describe here a protocol for the rapid processing of post-mortem autopsy tissue samples in order to generate durable patient-derived cell culture models that can be used in in vitro assays or in vivo orthotopic xenograft experiments. These models can be used to screen for potential drug targets and to study fundamental pathobiological processes within DIPG. This protocol can further be extended to analyze and isolate tumor and microenvironmental cells using Fluorescence-activated Cell Sorting (FACS), which enables subsequent analysis of gene expression, protein expression, or epigenetic modifications of DNA at the bulk cell or single cell level. Finally, this protocol can also be adapted to generate patient-derived cultures for other central nervous system tumors.

\section{Video Link}

The video component of this article can be found at https://www.jove.com/video/55360/

\section{Introduction}

DIPG is an aggressive central nervous system malignancy that arises in the ventral pons, typically during mid-childhood ${ }^{1,2}$. Despite decades of clinical trials, current treatment is limited to radiotherapy, which provides temporary improvement or stabilization of symptoms and only extends median survival by an average of 3 months. Even with radiotherapy, median overall survival is only 9 months with $90 \%$ of children dying from the disease within 2 years of initial diagnosis. Because of the infiltrative nature of the tumor and the critical functions of the brainstem, surgical resection is not possible. Moreover, in the United States, obtaining surgical biopsies for DIPG has historically not been performed ${ }^{3}$, as histopathological analysis has no current role in guiding clinical treatment and diagnosis can typically be made by neuroimaging alone. Thus, the availability of tumor tissue for study is restricted, limiting efforts to conduct research on candidate drug molecules and the underlying tumor biology. Notably, improvements in neuroimaging and stereotactic techniques have allowed for the development of safe biopsies of DIPG in recent years ${ }^{4}$, which, when combined with advancements in molecular biology, have transformed our understanding of the disease. Currently, multiple clinical trials using upfront biopsy and molecular profiling of DIPG for individualizing treatment plans are ongoing (NCT01182350, NCT02274987) ${ }^{5}$.

DIPG and other pediatric high-grade gliomas represent distinct diseases from their adult glioblastoma counterparts ${ }^{6,7}$, and thus faithful experimental models of DIPG are necessary to understand its unique pathophysiology and discover effective therapeutic strategies. In recent years, focused efforts to obtain DIPG tumor tissue for research at the time of autopsy or, less commonly, biopsy have revolutionized our understanding of and ability to study DIPG. Genome-wide sequencing efforts revealed a recurrent mutation in the $\mathrm{H} 3$ histone, family $3 \mathrm{~A}(\mathrm{H} 3 \mathrm{~F} 3 \mathrm{~A}$ ) and histone cluster $1, \mathrm{H} 3 \mathrm{~b}(H I S T 1 H 3 B)$, representing the first example of human disease caused by mutations in histone coding proteins ${ }^{8,9,10,11}$. Furthermore, a subset of DIPGs expresses mutations in the ACVR1 gene, which have not previously been reported in cancer but are identical to mutations found in the congenital childhood developmental disorder fibrodysplasia ossificans progressiva ${ }^{8,9,10,11}$. As well, the first patient-derived DIPG cell cultures and orthotopic xenograft models have now been established ${ }^{1,2,12,13,14}$, as well as mouse models established through the direct xenotransplantation of tumor cells into immunodeficient mice to generate serial xenografts ${ }^{3,14,15}$. Initial drug screening efforts using these patient-derived models have identified promising novel agents for clinical translation ${ }^{4,14}$ and have laid the groundwork for at least one clinical trial (NCT02717455).

These first steps towards progress are only the beginning, and numerous patient-derived tissue samples and culture models will be necessary to define subtypes of the disease and develop the most effective therapeutic strategies. Genetically engineered mouse models of DIPG will also facilitate studies aimed at furthering our basic understanding of the disease. Efforts made to generate genetic models for DIPG will be aided by the foundational genomic studies discussed above, but presently a limited number of options are available. One such model, which generates histologically similar high-grade brainstem gliomas, uses the viral RCAS/tv-a system to drive PDGF-B overexpression and Ink4a-ARF 
loss in nestin-positive cells in the posterior fossa of neonatal mice ${ }^{5,16}$. Another approach involves recapitulating several major DIPG-associated mutations (histone H3.3 K27M mutation, p53 loss and PDGFRA activation) in neural precursor cells derived from human embryonic stem cells, which is sufficient to render these cells tumorigenic ${ }^{6,7,17}$. The combination of genetic methods and patient-derived models will enable preclinical testing and further the development of effective therapies.

To address the challenges to DIPG research detailed above, this rapid autopsy protocol was developed to generate patient-derived cell cultures for investigation $8,9,10,11,12,14$. The protocol is intended to protect the viability of the tissue and maximize the likelihood of generating durable cultures despite the challenges associated with extended post-mortem intervals and transportation time. When generated successfully, these DIPG cultures can be used in subsequent in vitro manipulations and in vivo xenograft experiments, allowing for evaluation of potential therapeutic molecules on patient-derived cells.

\section{Protocol}

This protocol has been performed with approval from our institutional review board with appropriate de-identification of all patient data and in accordance to all institutional, national, and international guidelines for human welfare. Obtain all appropriate institutional review board approval and informed consent from the patient's family prior to working with this protocol.

\section{Obtaining Tissue Samples}

NOTE: A critical component of this protocol requires proper handling of the tissue donation starting immediately at the time of autopsy. The overall sample viability depends significantly on minimizing the Post-Mortem Interval (PMI), immediately transferring the tissue into the appropriate cold medium supplemented with antibiotics/antimycotics, keeping the sample on wet ice throughout transportation, and maintaining sterile technique throughout the protocol.

1. Upon notification of an imminent donation, prepare a sample collection kit. Using a shipping box with an insulated container that can be directly used for return transportation of the tissue sample, include the following materials:

1. Include materials for sterile preparation, including sterile gloves, drapes, and scalpels.

2. Include 8 - 10 sterile $50 \mathrm{~mL}$ conical tubes containing $30 \mathrm{~mL}$ shipping media in the insulated container with ice or cold packs. NOTE: While any standard cell culture media can work, the best sample viability is obtained with Hibernate-A supplemented with antibiotic/antimycotic.

3. Include any further sample tubes for other analyses (e.g., fixatives).

4. If the autopsy will not be performed at the investigator's site, include a document that clearly states how to collect the tumor sample. This includes details such as maintaining sterility, keeping sample tubes on wet ice, and including samples from the midbrain and medulla as tumor cells often invade these regions.

2. Maintain sterility during the autopsy. Consider the brain sterile inside the cranium, so observe sterile technique (including changing gloves) once the cranium is open. Avoiding contamination with skin and hair is critical.

3. Dissect the tumor from the ventral side (the "belly" of the pons, see Supplemental Figure 1). With a sterile scalpel, cut small $1 \mathrm{~cm}$ chunks from the tumor and immediately transfer into cold shipping media (see NOTE in step 1.1.2) and put on wet ice. Place $10 \mathrm{~mL}$ of tissue into each tube, resulting in a final volume of tissue and media in each tube of $40 \mathrm{~mL}$.

NOTE: In the case of DIPG, the tumor diffusely infiltrates the pons and frequently the rest of the brainstem. As such, collect as much of the sample as possible, typically including $3-4$ tubes ( $30-40 \mathrm{~mL}$ tissue) from pons and $1-2$ tubes $(10-20 \mathrm{~mL}$ tissue) each from midbrain and medulla.

4. Collect samples from the midbrain and medulla juxtaposed to the pons, which often contain many tumor cells.

5. After sample collection, ship the samples $\mathrm{O} / \mathrm{N}$ on wet ice in the insulated container. Do not ship the sample on dry ice, as freezing will kill the cells.

\section{Preparation for Sample Processing}

1. Prior to beginning sample preparation, sterilize tissue culture hoods along with razor blades, curved hemostats, and any other non-sterile tools under UV light for $1 \mathrm{~h}$. Perform all of the following steps under sterile conditions to prevent contamination of the cultures.

2. Prepare and sterile filter solutions.

1. To prepare $1.8 \mathrm{M}$ sucrose solution, dissolve $308.07 \mathrm{~g}$ sucrose in $300 \mathrm{~mL}$ distilled water. Add $50 \mathrm{~mL} 10 \mathrm{x}$ Hank's-buffered Saline Solution (HBSS) without calcium or magnesium and bring total volume to $500 \mathrm{~mL}$ using distilled water. Store at $4{ }^{\circ} \mathrm{C}$.

2. To prepare enzymatic digestion solution, take $50 \mathrm{~mL}$ HBSS with calcium and magnesium for every $10 \mathrm{~mL}$ of minced tissue and add $500 \mu \mathrm{L} 5 \mathrm{mg} / \mathrm{mL}$ deoxyribonuclease I (final concentration $50 \mu \mathrm{g} / \mathrm{mL}$ ), $500 \mu \mathrm{L} 2.5 \mathrm{mg} / \mathrm{mL}$ collagenase I/II and dispase solution (final concentration $25 \mu \mathrm{g} / \mathrm{mL}$ ), and $500 \mu \mathrm{L} 1 \mathrm{M} \mathrm{HEPES}$ buffer. Prewarm digestion solution in a $37^{\circ} \mathrm{C}$ water bath.

3. To prepare tumor stem media (TSM) base, mix $250 \mathrm{~mL}$ Neurobasal-A, $250 \mathrm{~mL}$ Dulbecco's Modified Eagle Medium: Nutrient Mixture F12 (DMEM/F12), $5 \mathrm{~mL}$ 100x antibiotic-antimycotic, $5 \mathrm{~mL} 200 \mathrm{mM} \mathrm{L}$-alanyl-L-glutamine dipeptide (GlutaMAX-A), $5 \mathrm{~mL}$ HEPES buffer, 5 $\mathrm{mL} 100 \mathrm{mM}$ sodium pyruvate, and $5 \mathrm{~mL} 100 x$ MEM non-essential amino acids.

4. To prepare complete TSM with growth factors, add the following supplements to TSM base: 50x B27 Supplement Minus Vitamin A (1:50), human Epidermal Growth Factor (H-EGF, $20 \mathrm{ng} / \mathrm{mL}$ ), human Fibroblast Growth Factor (H-FGF, $20 \mathrm{ng} / \mathrm{mL}$ ), human Plateletderived Growth Factor AA (H-PDGF-AA, $10 \mathrm{ng} / \mathrm{mL}$ ), human Platelet-derived Growth Factor BB (H-PDGF-BB, $10 \mathrm{ng} / \mathrm{mL})$, and heparin solution $(2 \mu \mathrm{g} / \mathrm{mL})$.

3. Precool a laboratory centrifuge equipped with a swinging-bucket rotor to $4{ }^{\circ} \mathrm{C}$. 


\section{Mechanical Dissociation}

1. Transfer the tissue into a high-walled $100 \mathrm{~mm} \times 20 \mathrm{~mm}$ cell culture dish. Remove media leftover from shipping and replace with $10-15 \mathrm{~mL}$ cold culture media.

2. Using the curved hemostats to grasp a razor blade, mince the tissue finely while removing obvious blood vessels or meninges. NOTE: The final tissue fragments should be smaller than $1 \mathrm{~mm}$ (see Figure 1B).

3. Transfer the tissue into a clean $50 \mathrm{~mL}$ conical tube. Wash the cell culture dish with an additional $5 \mathrm{~mL}$ cold culture media and transfer to the conical tube. Repeat this wash step as necessary to transfer remaining tissue.

4. Using a $10 \mathrm{~mL}$ serological pipet, triturate gently (4 - 5 times). Allow larger tissue fragments to settle to the bottom of the tube. If necessary, briefly centrifuge the sample for $1 \mathrm{~min}\left(350 \mathrm{xg}, 4^{\circ} \mathrm{C}\right)$.

5. Collect the mechanically dissociated fraction.

1. Remove the supernatant and filter it through a $100 \mu \mathrm{m}$ filter into a new $50 \mathrm{~mL}$ conical tube labeled "Mechanical Dissociation." Invert the filter over the original conical tube and wash with culture media to recover tissue fragments.

2. Centrifuge the "Mechanical Dissociation" fraction for $5 \min \left(350 \times \mathrm{g}, 4^{\circ} \mathrm{C}\right)$.

3. Remove the supernatant from the pelleted "Mechanical Dissociation" fraction and resuspend the tissue in cold culture media. If there is more than $5 \mathrm{~mL}$ of tissue in the "Mechanical Dissociation" conical tube, split the tissue into other $50 \mathrm{~mL}$ conical tubes such that no tube has more than $5 \mathrm{~mL}$ tissue.

6. Store the mechanically dissociated fraction on ice until the sucrose gradient centrifugation step (Step 5). Alternatively, the mechanically dissociated fraction can proceed to Step 5 during the enzymatic dissociation incubation period (Step 4.4).

\section{Enzymatic Dissociation}

1. If there is more than $5 \mathrm{~mL}$ of tissue in the tube containing the remaining larger tissue fragments, split the tissue into other new $50 \mathrm{~mL}$ conical tubes such that no tube has more than $5 \mathrm{~mL}$ tissue.

2. Centrifuge the conical tube(s) containing the remaining tissue fragments for 5 min $\left(350 \times \mathrm{g}, 4^{\circ} \mathrm{C}\right)$.

3. Remove the supernatant and add the prewarmed enzymatic digestion solution, such that there is $5 \mathrm{~mL}$ digestion solution for every $1 \mathrm{~mL}$ tissue (e.g., $25 \mathrm{~mL}$ digestion solution for $5 \mathrm{~mL}$ tissue).

4. Seal the conical tube lids with laboratory film and incubate the reaction on a rotator at $37^{\circ} \mathrm{C}$ for $30 \mathrm{~min}$.

5. After the incubation, triturate the samples gently (see Figure 1C).

1. Using a $10 \mathrm{~mL}$ serological pipet, pipet the sample up and down 6 - 8 times. Avoid generating excessive air bubbles.

2. Add a $1,000 \mu \mathrm{L}$ pipet tip to the end of the pipet and triturate an additional 6 - 8 times.

3. Allow any remaining chunks to settle to the bottom of the tube. Remove and filter the supernatant with the cells still suspended through a $100 \mu \mathrm{m}$ filter into a new $50 \mathrm{~mL}$ conical tube labeled "Enzymatic Dissociation" and store on ice.

4. If significant tissue fragments remain, add an additional $10 \mathrm{~mL} \mathrm{HBSS}$ with calcium and magnesium to the fragments and repeat steps 3.5.1 and 3.5.2. Filter this final solution through a $100 \mu \mathrm{m}$ filter into the "Enzymatic Dissociation" tube.

6. Centrifuge the "Enzymatic Dissociation" tube for $5 \min \left(350 \mathrm{xg}, 4^{\circ} \mathrm{C}\right)$ and continue to sucrose gradient centrifugation.

\section{Sucrose Gradient Centrifugation}

1. If samples are still suspended in solution, centrifuge for $5 \min \left(350 \times \mathrm{g}, 4{ }^{\circ} \mathrm{C}\right)$.

2. Remove the supernatant and resuspend tissue in $20 \mathrm{~mL}$ cold HBSS without calcium and magnesium. Bring volume up to $25 \mathrm{~mL}$ total with cold HBSS.

3. Slowly add $25 \mathrm{~mL} 1.8 \mathrm{M}$ sucrose solution and invert the tube to mix. This results in a $0.9 \mathrm{M}$ sucrose gradient.

4. Centrifuge with no brake for $10 \mathrm{~min}\left(800 \mathrm{xg}, 4^{\circ} \mathrm{C}\right)$. Using a centrifugation brake will disrupt the gradient and reduce yield (see Figure 1D for an example of a sample before and after centrifugation).

5. Carefully aspirate myelin debris and as much sucrose solution as possible. Wash the sample by adding $30 \mathrm{~mL}$ cold HBSS without calcium and magnesium and mixing gently. Centrifuge for $5 \min \left(350 \times \mathrm{g}, 4^{\circ} \mathrm{C}\right)$.

\section{ACK Red Blood Cell Lysis}

1. Remove the wash supernatant. Add $5 \mathrm{~mL}$ ACK lysis buffer and gently resuspend the cell pellet, swirling the tube for $1 \mathrm{~min}$ at RT.

2. Quench the lysis by adding $30 \mathrm{~mL}$ cold HBSS without calcium and magnesium.

3. Centrifuge for $5 \min \left(350 \times \mathrm{g}, 4^{\circ} \mathrm{C}\right)$.

\section{Initial Culture Maintenance}

1. Resuspend the final cell pellets in $10-15 \mathrm{~mL}$ warm complete TSM with growth factors and quantify viable cell density on a hemocytometer using trypan blue exclusion.

NOTE: The range of viable cells varies widely depending on the circumstances of the tissue donation, and quantification can be difficult at this early stage due to leftover cellular debris. In an ideal case, aim to have one million live cells per sample. In cases where excessive debris remains, plate at a lower density in a T175 flask.

2. Transfer the final cell suspensions to a new T75 culture flask. Spike in additional growth factors every other day to maintain overall growth factor levels, and monitor for development of tumor cell neurospheres (see Figure 1E and Figure 2).

3. Alternatively, process enzymatically dissociated cells for further analysis, including flow cytometry and fluorescence-activated cell sorting. 
4. After the development of neurospheres (which on average takes $3-4$ weeks, but ranges from a few days to as long as 2 months), reverse filter the sample using a $100 \mu \mathrm{m}$ nylon mesh filter to reduce the amount of debris.

NOTE: The filtrate should be maintained in culture in case viable single cells or small spheres are present.

5. Ensure the integrity and purity of the culture by performing DNA fingerprinting while passaging, comparing to the initial patient sample.

\section{Representative Results}

The protocol described is summarized as a five-step workflow with images of the tissue at various stages of processing in Figure 1. The sample is first obtained through rapid sterile autopsy. During mechanical dissociation, the tissue is minced while removing blood vessels and meninges from the sample, and filtered through a $100 \mu \mathrm{m}$ nylon mesh filter. Remaining tissue fragments are enzymatically dissociated in a warming oven. Next, debris is reduced through sucrose gradient centrifugation, isolating a distinct layer of myelin from the sample. In addition, ACK lysis visibly reduces the amount of red blood cells present in the sample. Finally, the cells are plated in serum-free media supplemented with growth factors.

Supplemental Figure 1 is an example of the tumor upon autopsy. The tumor grows as a diffuse infiltration of the pons and the adjoining regions of the brainstem. During sample preparations, small $\sim 1 \mathrm{~cm} \times 1 \mathrm{~cm}$ chunks should be cut and immediately placed into cold shipping media on ice.

Figure 2 shows various examples of how the samples can appear from the early stages of culturing to a durable culture. Initially plated and early cultures (A, B) often do not appear to contain many surviving cells. Furthermore, early cell clusters (C, D) often do not exhibit the degree of contrast typically associated with neurospheres. Notably, the duration of time in culture prior to the appearance of cell clusters can take weeks to a couple months. However, the initial passage of these cell clusters, combined with reverse filtering of cell clusters, can isolate healthy tumor cells that are able to form spheres $(F)$. The filtrate $(E)$ typically contains leftover debris; however, we recommend plating and maintaining the filtrate and monitoring for the development of cell clusters. These patient-derived samples can then be maintained in culture for multiple passages $(G, H)$.

Figure 3 demonstrates the ability of these cells to be xenografted into mice. In each of these cases, the DIPG cells were transfected with a GFPLuciferase reporter to monitor the development of the tumors through bioluminescence (A). The tumors can also be examined histologically, for example to observe tumor engraftment (B) or expression of specific markers (C). These in vivo models, combined with in vitro assays can be used to assess the effects of various drugs or gene manipulations (e.g., $8,9,10,11,14$ ). 


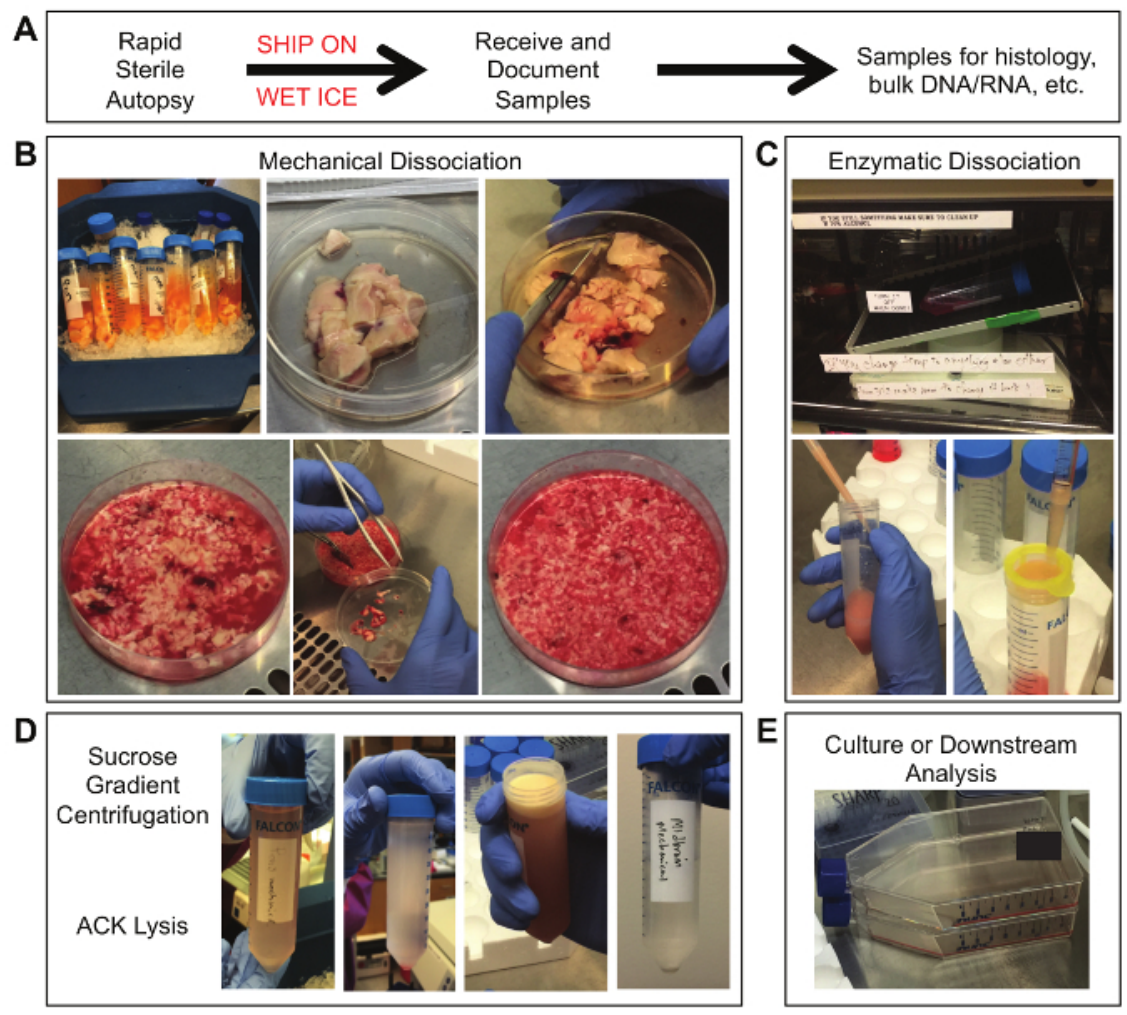

Figure 1. Tissue Samples at Various Stages of Processing. (A) Obtain the sample through sterile autopsy procedures and overnight shipping on wet ice. (B) From left to right: (row 1) tubes containing tumor tissue in shipping media; fresh tissue in a $100 \mathrm{~mm} \times 20 \mathrm{~mm}$ cell culture dish; initial mincing of tissue; (row 2) partially minced tissue; removal of meninges and blood vessels; final size of minced tissue pieces. (C) From left to right: (row 1) tissue incubated on a rotating table in a $37^{\circ} \mathrm{C}$ oven; (row 2) trituration of tissue through a $1000 \mu \mathrm{L}$ pipet tip attached to a $10 \mathrm{~mL}$ pipet; filtering of dissociated tissue through a $100 \mu \mathrm{m}$ nylon mesh filter. (D) From left to right: dissociated tissue suspended in $0.9 \mathrm{M}$ sucrose solution prior to centrifugation; dissociated tissue separated across a sucrose gradient after centrifugation; a visible layer of myelin debris on top of the sucrose gradient; a final cell pellet after ACK lysis and wash. (E) The final sample can be placed into culture or used in other downstream analyses. Please click here to view a larger version of this figure. 

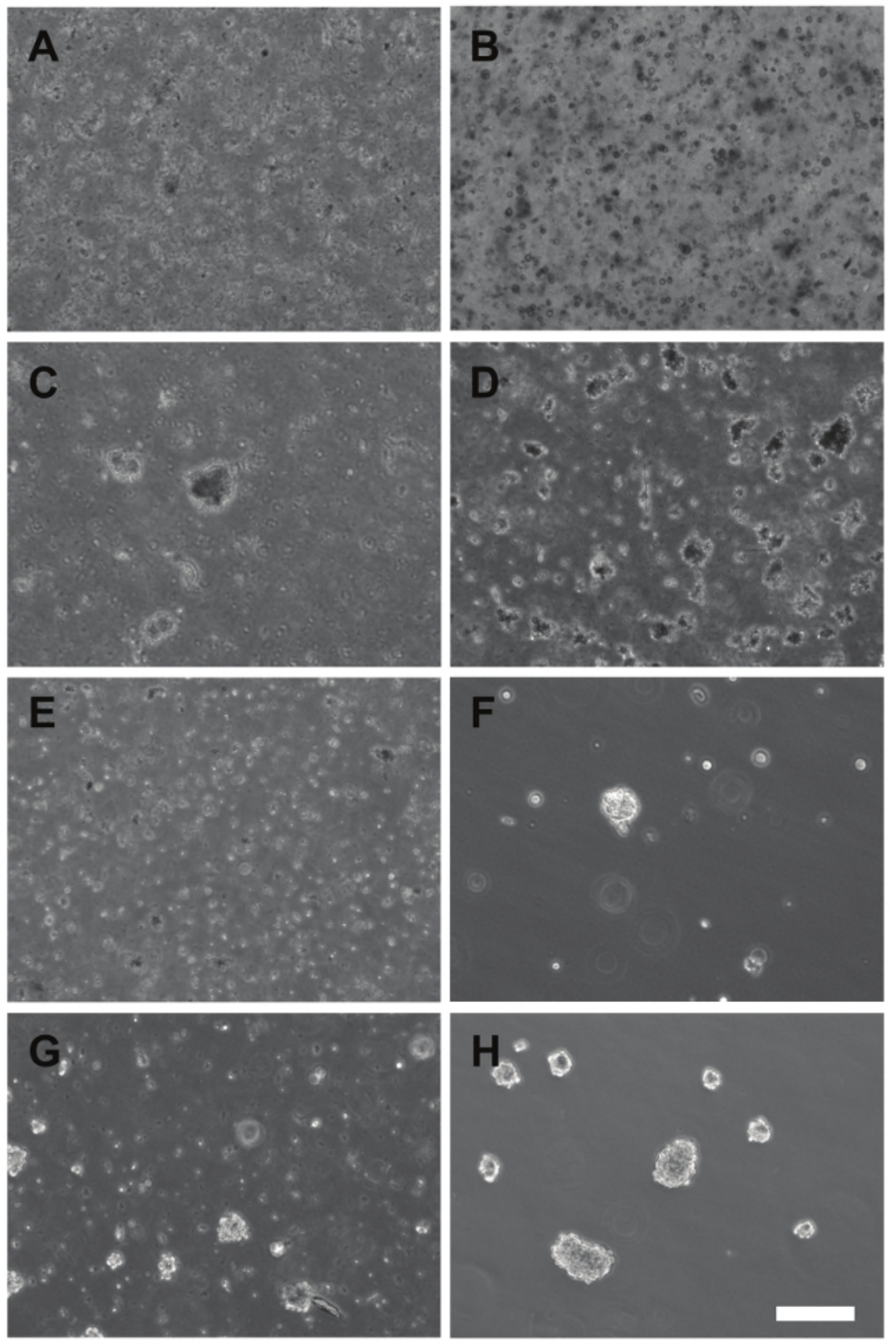

Figure 2. Images of Primary Cultures and Early Passage Cells. (A - B) Cultures plated immediately after dissociation (SU-DIPG-XXX, A), or that have not grown neurospheres yet (SU-DIPG-XXIX, B). (C - D) Early appearance of neurospheres in primary cultures of SU-DIPG-XXVIII (C) and SU-DIPG-XXIX (D). (E-F) First passage of the primary culture from D using a $100 \mu \mathrm{m}$ nylon filter, with the filtrate $(E)$ and reverse filtrate $(F)$ plated. $(\mathbf{G}-\mathbf{H})$ Mature neurospheres from the first passage of SU-DIPG-XXVII (G) and the third passage of SU-DIPG-XXV (H) growing in culture. Scale bar $=200 \mu \mathrm{m}$. Please click here to view a larger version of this figure. 
A
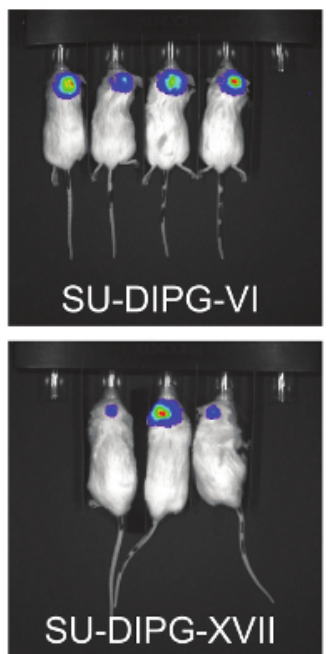
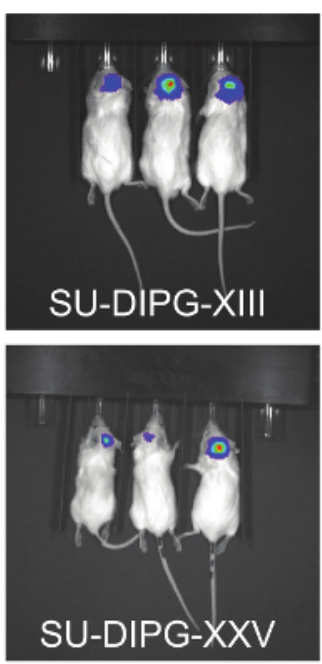

B

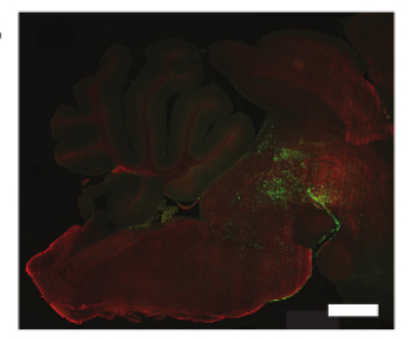

C

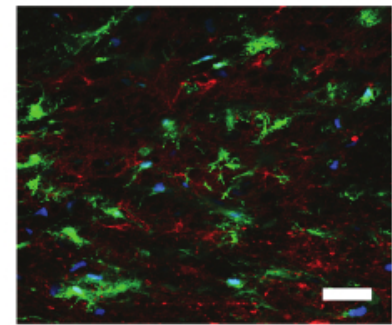

Figure 3. Patient-derived Cell Cultures Can Engraft and Form Tumors in Mice. (A) Bioluminescence images of four different patient-derived cell cultures transfected with a GFP-Luciferase reporter. (B) Sagittal section from a mouse xenograft, showing tumor cells engrafted in the mouse pons. Green: GFP, Red: Myelin basic protein. Scale bar $=1 \mathrm{~mm}$. (C) Example immunofluorescence image showing GFP tumor cells engrafted in a mouse brain. Blue: DAPI, Green: GFP, Red: IGF2R. Scale bar $=40 \mu \mathrm{m}$. Please click here to view a larger version of this figure.

Supplemental Figure 1. Autopsy Sample of a Pontine Tumor. Immediate post-mortem appearance of a diffuse intrinsic pontine glioma. The tumor appears as a large, myelin-rich mass on the ventral surface of the pons. Please click here to download this image.

\section{Discussion}

This protocol describes a method for the rapid processing of post-mortem tumor tissue donations to develop patient-derived cell cultures, which can be used in a variety of in vitro or in vivo experiments. Because of the nature of working with autopsy samples, maintaining sample viability poses a significant challenge. Several contributing factors, including the post-mortem interval to autopsy or the time required for transportation of tissue, should be minimized as much as possible but often are hard to control. In our experience, a postmortem interval of less than 6 - $8 \mathrm{~h}$ (from the time of death to the time that the tissue is placed in ice cold shipping and transportation media) has yielded the best chance of establishing a durable cell culture. It is best to then receive the tissue in the lab within $24 \mathrm{~h}$, and process it for culture immediately upon receipt.

Since the location of these rare cases varies widely, and the quality of the tissue retrieval step strongly affects the chance of success, the logistics of performing the autopsy can be challenging. In many cases, in order to achieve the 6-8 hour time frame, it is not feasible to conduct the tissue harvest at an academic center. Instead, having a tissue recovery service on call to recover the tumor tissue at the funeral home under an IRB-approved protocol is often more expedient for tissue recovery. Obtaining informed consent in advance of death is recommended and facilitates the logistical planning. Preparing self-contained autopsy kits with clear and thorough instructions and sterile supplies for tissue retrieval (gloves, drapes, scalpels) is strongly recommended. Additionally, establishing clear points of communication amongst the investigator, pathologist, and funeral home is critical. For example, the investigator should discuss the protocol with the pathologist prior to the autopsy and highlight common errors. These errors include microbial contamination (usually yeast) during tissue retrieval, failure to ship through overnight/ expedited shipping and failure to ship the sample on sufficient wet ice in a thermoinsulated container. Finally, we recommend using a door-todoor courier service for shipment of the sample to minimize transportation time.

Care should also be taken during tissue dissociation to preserve cell viability. For instance, the use of media designed to preserve neural tissue health for transport (Hibernate-A supplemented with antibiotic/antimycotic) will increase the chance of generating a successful culture. It is also important to keep the sample at a cold temperature throughout the protocol by always transferring the samples on ice. Collecting both mechanical and enzymatic dissociation fractions along with minimizing trituration can improve protocol success, as both steps are traumatic to cells. In our experience, while most successful cultures grow out of both fractions, we have had instances where only one of the two preparations established a durable culture, potentially reflecting the delicate nature of these samples. Another traumatic step in the protocol is trituration; one strategy that can reduce the degree of trituration necessary is to ensure the samples are thoroughly minced at the very beginning of the preparation. Other examples of aspects of the protocol designed to improve the sample viability include adding buffers (e.g., HEPES) throughout the protocol, which is especially critical during enzymatic dissociation.

A possible modification of this protocol to further increase cell viability is the removal of the ACK red blood cell lysis step. However, in this case, it is often then necessary to perform an ACK lysis step during the initial week in culture to remove red blood cells. Another aspect of this protocol that may be modified is the elimination of myelin and cellular debris using a sucrose density gradient. Specifically, other strategies that have been reported to improve cell viability of microglial cells are also possible at this step, such as a discontinuous Percoll density gradient ${ }^{18}$ or magnetic myelin removal beads.

Finally, the duration between initial tissue dissociation and the appearance of neurospheres varies between samples and can take anywhere from a week to a month or more. Since the initial cultures typically contain a significant degree of dead cells and cellular debris, it can be challenging to determine whether viable cells remain; the culture should be maintained for at least 4 - 8 weeks (Figure 2). One strategy for isolating growing cells from the remaining debris is presented here (i.e. reverse filtering cell clusters and re-plating in fresh media). The decision 
about whether to continue to maintain a culture is ultimately a case-by-case decision based upon factors surrounding the autopsy (e.g., a prolonged postmortem interval, issues during tissue transport), the tissue dissociation (e.g., excessive blood or debris), and how the sample appears in culture. Once a culture is established, the identity should be validated by DNA fingerprinting using short tandem repeat analysis or a similar method, comparing the culture to a sample of the original tumor retained for this purpose. We recommend routinely validating cultures by DNA fingerprinting to ensure that no contamination by other cultures has occurred, for example every 3 - 6 months.

The generation of these patient-derived DIPG cultures represents an important step in the successful development of effective therapies. The expansion of the available patient-derived DIPG cultures and xenograft models will be critical to identifying the most effective therapeutic strategies and ultimately overcoming this devastating disease.

\section{Disclosures}

The authors have nothing to disclose.

\section{Acknowledgements}

The authors gratefully acknowledge support from the McKenna Claire Foundation, National Institute of Neurological Disorders and Stroke (NINDS K08NS070926 and R01NS092597), Department of Defense (NF140075), California Institute for Regenerative Medicine (CIRM RB4-06093 and RN3-06510), Alex's Lemonade Stand Foundation, The Cure Starts Now Foundation and DIPG Collaborative, Lyla Nsouli Foundation, Unravel Pediatric Cancer, Childhood Brain Tumor Foundation, Matthew Larson Foundation, V Foundation, Godfrey Family Fund in Memory of Fiona Penelope, the Wayland Villars DIPG Foundation, the Dylan Jewett, Connor Johnson, Zoey Ganesh, Dylan Frick, Abigail Jensen, and Jennifer Kranz Memorial Funds, N8 Foundation, Virginia and D.K. Ludwig Fund for Cancer Research, Child Health Research Institute at Stanford Anne T. and Robert M. Bass Endowed Faculty Scholarship in Pediatric Cancer and Blood Diseases.

\section{References}

1. Fisher, P. G., et al. A clinicopathologic reappraisal of brain stem tumor classification. Identification of pilocystic astrocytoma and fibrillary astrocytoma as distinct entities. Cancer. 89 (7), 1569-1576 (2000).

2. Johung, T. B., \& Monje, M. Diffuse Intrinsic Pontine Glioma: New Pathophysiological Insights and Emerging Therapeutic Targets. Curr. Neuropharmacol. (2016).

3. Cage, T. A., et al. Feasibility, safety, and indications for surgical biopsy of intrinsic brainstem tumors in children. Child Nerv. Syst. 29 (8), 1313-1319 (2013).

4. Puget, S., et al. Biopsy in a series of 130 pediatric diffuse intrinsic Pontine gliomas. Child Nerv. Syst. 31 (10), 1773-1780 (2015).

5. Kieran, M. W. Time to rethink the unthinkable: Upfront biopsy of children with newly diagnosed diffuse intrinsic pontine glioma (DIPG). Pediatr. Blood Cancer. 62 (1), 3-4 (2014).

6. Sturm, D., et al. Paediatric and adult glioblastoma: multiform (epi)genomic culprits emerge. Nat. Rev. Cancer. 14 (2), $92-107$ (2014).

7. Jones, C., \& Baker, S. J. Unique genetic and epigenetic mechanisms driving paediatric diffuse high-grade glioma. Nat. Rev. Cancer. (2014).

8. Taylor, K. R., et al.Recurrent activating ACVR1 mutations in diffuse intrinsic pontine glioma. Nat. Genet. 46 (5), $457-461$ (2014).

9. Wu, G., et al.The genomic landscape of diffuse intrinsic pontine glioma and pediatric non-brainstem high-grade glioma. Nat. Genet. 46 (5), 444-450 (2014).

10. Fontebasso, A. M., et al. Recurrent somatic mutations in ACVR1 in pediatric midline high-grade astrocytoma. Nat. Genet. 46 (5), $462-466$ (2014)

11. Buczkowicz, P., et al. Genomic analysis of diffuse intrinsic pontine gliomas identifies three molecular subgroups and recurrent activating. Nat. Genet. 46 (5), 451-456 (2014).

12. Monje, M., et al. Hedgehog-responsive candidate cell of origin for diffuse intrinsic pontine glioma. Proc. Natl. Acad. Sci. 108 (11), $4453-4458$ (2011).

13. Aoki, Y., et al. An experimental xenograft mouse model of diffuse pontine glioma designed for therapeutic testing. J. Neuro-Oncol. 108 (1), 29-35 (2012).

14. Grasso, C. S., et al. Functionally defined therapeutic targets in diffuse intrinsic pontine glioma. Nat. Med., 1-10 (2015).

15. Shu, Q., et al.Direct Orthotopic Transplantation of Fresh Surgical Specimen Preserves CD133 + Tumor Cells in Clinically Relevant Mouse Models of Medulloblastoma and Glioma. Stem Cells. 26 (6), 1414-1424 (2008).

16. Becher, O. J., et al. Preclinical evaluation of radiation and perifosine in a genetically and histologically accurate model of brainstem glioma. Cancer Res. 70 (6), 2548-2557 (2010).

17. Funato, K., Major, T., Lewis, P. W., Allis, C. D., \& Tabar, V. Use of human embryonic stem cells to model pediatric gliomas with H3.3K27M histone mutation. Science. 346 (6216), 1529-1533 (2014).

18. Olah, M., et al. An optimized protocol for the acute isolation of human microglia from autopsy brain samples. Glia. 60 (1), $96-111$ (2012). 